\title{
Experimental analysis of true left-handed behaviour and transmission properties of composite metamaterials
}

\author{
Kaan Guven ${ }^{\text {a,* }}$, Koray Aydin ${ }^{\text {a }}$, Ekmel Ozbay ${ }^{\text {a,b }}$ \\ ${ }^{a}$ Department of Physics, Bilkent University, Bilkent, 06800 Ankara, Turkey \\ ${ }^{\mathrm{b}}$ Advanced Research Laboratory, Bilkent University, Bilkent, 06800 Ankara, Turkey
}

Received 18 August 2005; received in revised form 14 September 2005; accepted 16 September 2005

Available online 10 October 2005

\begin{abstract}
We report the true left-handed transmission of a composite metamaterial (CMM) consisting of periodically stacked split-ring resonator (SRR) and wire elements. The negative permeability $(\mu<0)$ gap is demonstrated explicitly by comparing SRR and closed-ring resonator structures. We confirm experimentally that the plasma cut-off frequency of the CMM is determined by the combined dielectric response of SRR and wire elements, and it is much lower than that of the wire-only medium. This is crucial to identify the left-handed transmission bands of the CMM. We further investigate the effect of intralayer and interlayer disorder on the transmission spectrum of CMM arising from misaligned fabrication and stacking of the SRR layers. We found that the intralayer disorder affects the $\mu<0$ gap of SRRs and the left-handed transmission band of CMM significantly, whereas the SRR transmission is rather immune to interlayer disorder.
\end{abstract}

(C) 2005 Elsevier B.V. All rights reserved.

PACS: 41.20.Jb; 78.20.Ci; 42.70.-a; 81.05.-t

Keywords: Left-handed material; Negative refraction; Split ring resonator

\section{Analysis of left-handed behaviour in composite metamaterials}

The intriguing physics of a medium having a negative refractive index was discussed by Veselago theoretically almost four decades ago [1]. Recently, this idea was brought to realization by means of a composite metamaterial (CMM), consisting of two components that have negative permittivity, $\varepsilon(\omega)$, and negative permeability, $\mu(\omega)$, simultaneously over a frequency range $[2,3]$. This was accomplished by periodic wire structures with $\varepsilon(\omega)<0$ for $\omega<\omega_{\mathrm{p}}$ (plasma frequency), and split-ring resonator (SRR) structures with $\mu(\omega)<0$ for $\omega \approx \omega_{\mathrm{m}}$ (magnetic resonance frequency) [4,5].

\footnotetext{
* Corresponding author. Tel.: +90 312 2901971; fax: +90 3122664579 .

E-mail address: guven@fen.bilkent.edu.tr (K. Guven).
}

The main challenge was to obtain negative permeability at frequencies beyond $\mathrm{MHz}$ regime where no natural material is known to exist. These components are then stacked to form a CMM, where the structural elements (i.e., wire, SRR) are much smaller than the propagating wavelength. As a result, the electromagnetic wave "sees" an effective medium with $n(\omega)=$ $\sqrt{\varepsilon(\omega) \mu(\omega)}<0[3]$.

The existence of a passband of CMM within the common stop bands of SRR-only and wire-only media is intuitively considered evidence for left-handed behaviour. Here, we demonstrate that the electric response of the CMM differs substantially from that of the wire-only medium, due to the electric response contribution of SRRs as predicted theoretically [6]. In this respect, the decomposition of CMM into "pure" $\varepsilon(\omega)<0$ and $\mu(\omega)<0$ components is not possible. 

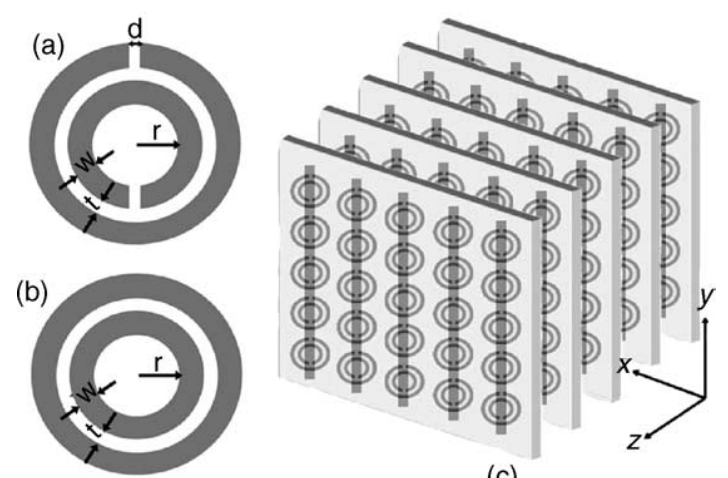

(c)

Fig. 1. The schematic representation of (a) SRR, (b) CRR, and (c) CMM structures. The parameters are $d=t=0.2 \mathrm{~mm}, w=0.9 \mathrm{~mm}$, $r=1.6 \mathrm{~mm}$. Wire thickness is $w=0.9 \mathrm{~mm}$. The unit cell has dimensions $a_{x}=a_{y}=8.8 \mathrm{~mm}$ and $a_{z}=6.5 \mathrm{~mm}$.

The total dielectric response of CMM can be obtained by incorporating a complementary structure of SRR, which exhibits only the isolated electric response. We accomplish this by introducing closed-ring resonators (CRR). By incorporating CRRs in the CMM (called CCMM) and determining the actual $\varepsilon(\omega)<0$ range, one can truly identify the passbands of CMM as being lefthanded or right-handed [7].

Before commencing the aforementioned analysis, let us summarize the fabrication of the structures and the measurement set-up: the SRR and CRR structures are fabricated on FR4 circuit board (1.6 mm thick, $\varepsilon=4.4)$ with $30 \mu \mathrm{m}$ deposited copper layer by etching. The geometrical parameters of SRR and CRR are $d=t=0.2 \mathrm{~mm}, w=0.9 \mathrm{~mm}, r=1.6 \mathrm{~mm}$ (see, Fig. 1). Wires are continuous along the board with a thickness of $w=0.9 \mathrm{~mm}$. The dimensions of a unit cell containing an SRR (wire) are $a_{x}=a_{y}=8.8 \mathrm{~mm}$ and $a_{z}=6.5 \mathrm{~mm}$. Each sample (SRR, wire, CMM, CCMM) has 5, 15, and 18 unit cells in the $x, y$, and $z$, directions, respectively.
The transmission spectrum is measured by using a network analyzer and two horn antennas. The incident field has wavevector $k$, electric field $E$, and magnetic field $H$, along the $x, y$, and $z$ directions, respectively.

In Fig. 2(a), we compare the transmission spectra of SRR and CRR structures. They exhibit similar electric response, but the SRR distinguishes by its magnetic response at $f \sim 3.8 \mathrm{GHz}$. The second stop band of SRR is not due to $\mu(\omega)<0$ behaviour. Fig. 2(b) shows that the total dielectric response of the CCMM $(\mathrm{CRR}+$ wire) is significantly lower from that of the wire-only medium. We see that the actual CMM (SRR + wire) in Fig. 2(c) has two transmission bands: the first band coincides with the stop band of SRR-only structure and still resides in the $\varepsilon(\omega)<0$ region, and hence identified as truly left-handed. In the second band, the CMM acts as an ordinary right-handed medium. If the analysis would have been conducted accoring to the electric response of the wires only, the transmission band of CMM could not be explained, since the CMM medium would have $\mu(\omega)>0$ and $\varepsilon(\omega)<0$.

To investigate the negative refraction by this CMM we constructed a prism-shaped 2D structure [8]. The unit cell of the 2D CMM prism, the photo of the prism structure, and the experimental set-up are shown in Fig. 3(a-c), respectively. The source is located at $\sim 2 \lambda$ away from the interface; the transmission is measured as a function of angle around the normal of the oblique interface at a distance $\sim 10 \lambda$, whereas the frequency is swept from 3.73 to $4.05 \mathrm{GHz}$. The angular refraction spectrum shown in Fig. 3(d) indicates that the beam refracts negatively for the entire passband. The refractive index has frequency dependent anisotropy indicated by the shift of the refracted beam profile with frequency. Fig. 3(d) also shows the angular beam profile at $f=3.92 \mathrm{GHz}$. Using Snell's Law
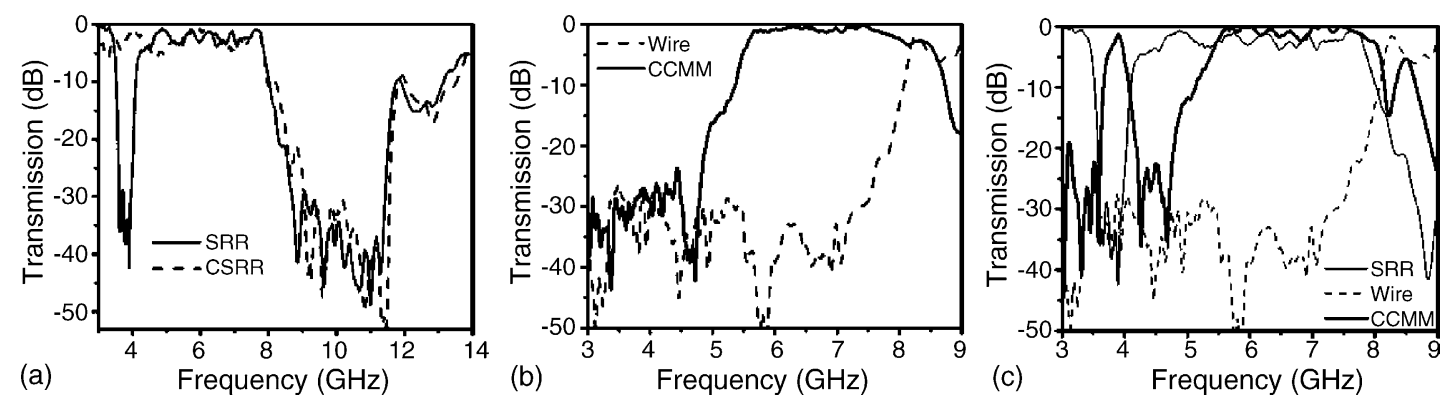

Fig. 2. (a) The transmission spectra of SRR (solid) and CRR (dashed) structures. Note the similar electric response around $10 \mathrm{GHz}$. Magnetic resonance of SRR is around $4 \mathrm{GHz}$. (b) Comparison of wire (dashed) and CCMM (CRR + wire) (solid) spectra showing $\omega_{\mathrm{p}}$ of wires ( $\sim 8 \mathrm{GHz}$ ) and that of CCMM ( $\sim 5 \mathrm{GHz})$. (c) Comparison of wire (dotted), SRR (thin), and CMM (thick solid) spectra. Only the first transmission band of CMM $(\sim 4 \mathrm{GHz})$ is left-handed. 


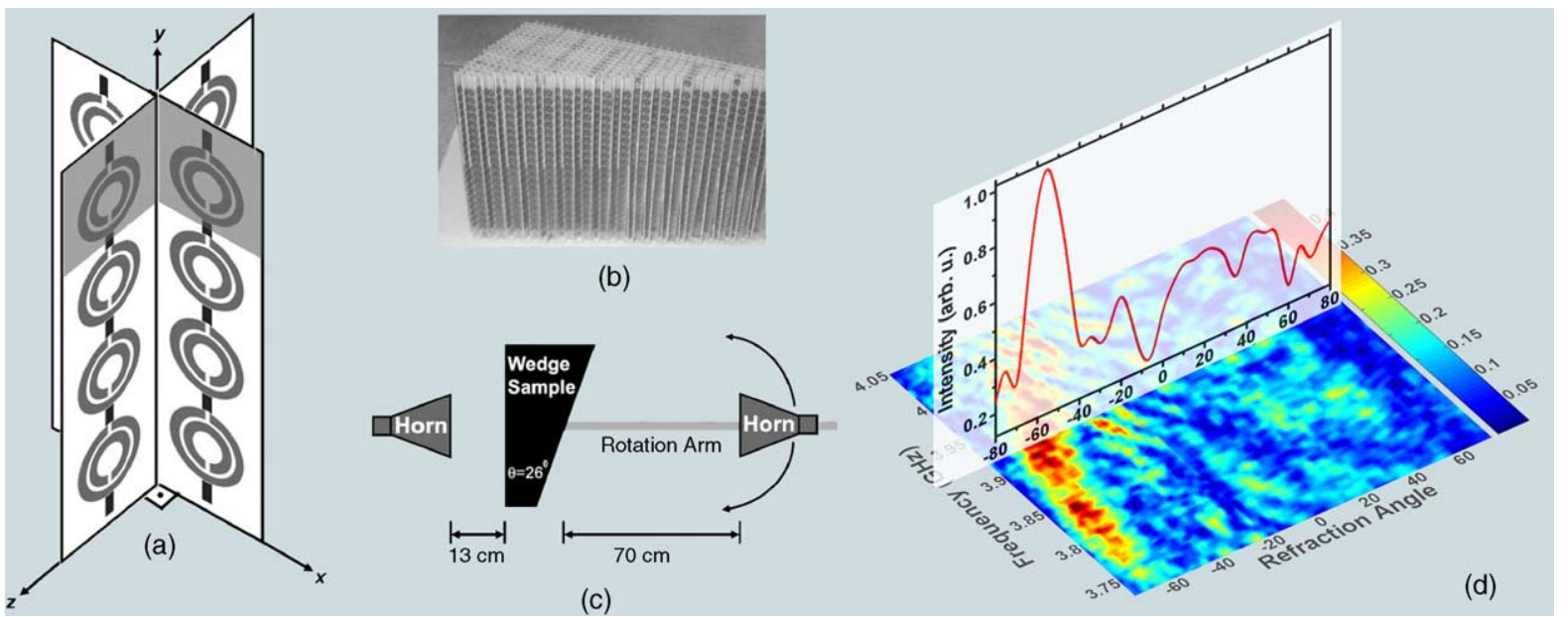

Fig. 3. (a) The unit cell (shaded) of the 2D CMM. (b) Actual photo of the $2 \mathrm{D}$ CMM prism with $\theta_{\mathrm{t}}=26^{\circ}$ apex angle. (c) Schematic of the experimental set-up. (d) The angular refraction spectrum. The light shaded band around $-60^{\circ}$ denotes higher intensity. The angular cross-section at $f=3.92 \mathrm{GHz}$ yields a refractive index $-1.87 \pm 0.05$.

$n_{\mathrm{CMM}} \sin \theta_{\mathrm{t}}=n_{\mathrm{AIR}} \sin \theta_{\mathrm{r}}$, with the experimental data $\left(\theta_{\mathrm{t}}=26^{\circ}, \theta_{\mathrm{r}}=-55^{\circ}\right)$, we obtain the refractive index to be $n_{\text {eff }}=-1.87 \pm 0.05$.

\section{Effect of disorder on the magnetic resonance and left-handed transmission of CMM structures}

Most of the experimental studies concerning CMMs are performed in the microwave regime, where the structures can be fabricated with high precision. However, in the infrared and optical regime, where submicron scale structures are needed, the disorder effects may become comparable to a characteristic length scale, and may turn out to be an intrinsic problem. By virtue of this fact, we artificially induce and investigate the disorder effects in the microwave regime [9]. Disorder can occur during the fabrication of SRR and wire patterns. The stacking method for constructing CMM is prone to misalignment. At present, we consider the disorder effects on SRR structures only. For wires, it is well known that the $\varepsilon<0$ behaviour is generated by the periodic alignment and any disorder reduces $\omega_{\mathrm{p}}$ [4].
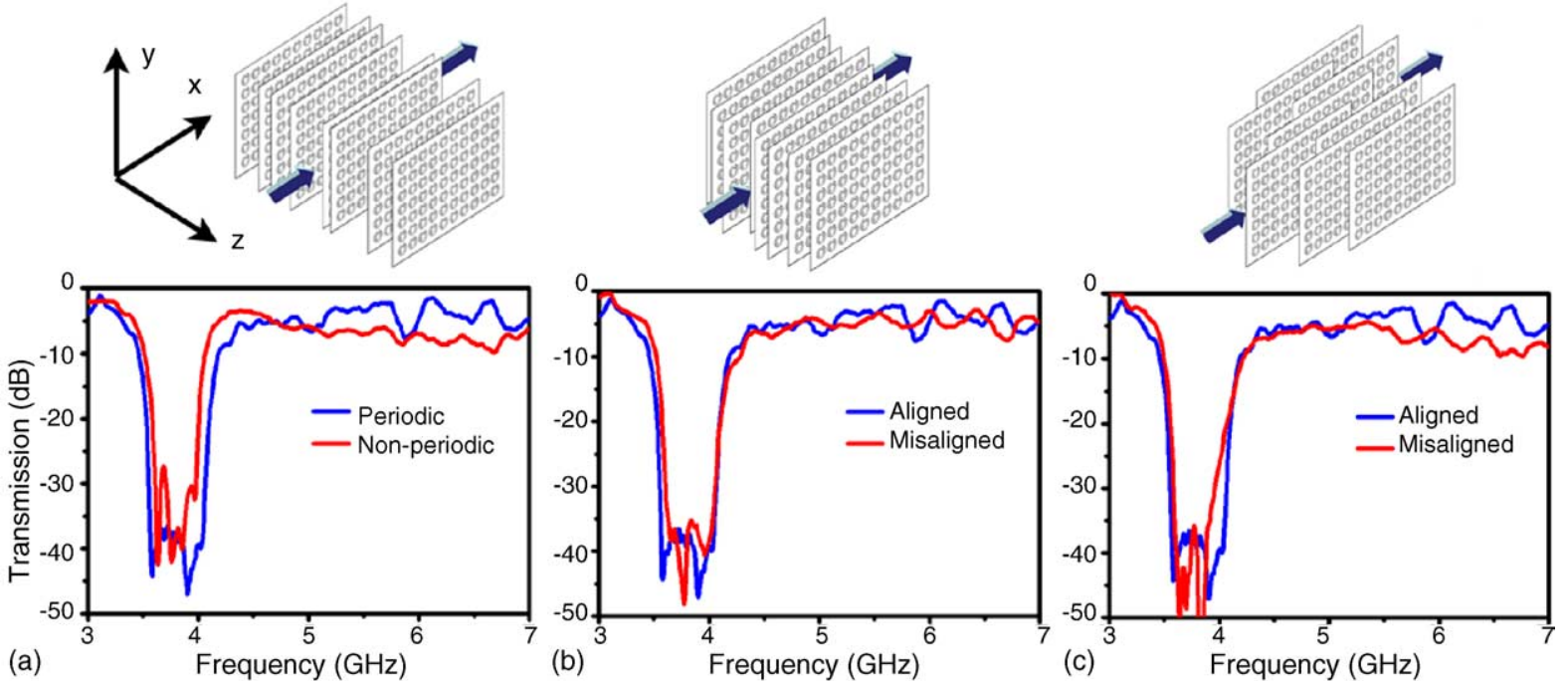

Fig. 4. (Top) The misalignment along (a) the lateral (z) and (b and c) the longitudinal ( $x$ ) directions, (b) weak misalignment, and (c) strong misalignment in $x$ direction. (Bottom) The transmission spectra (light lines) of the misaligned structures compared to that of the fully ordered SRR structure (dark lines). 

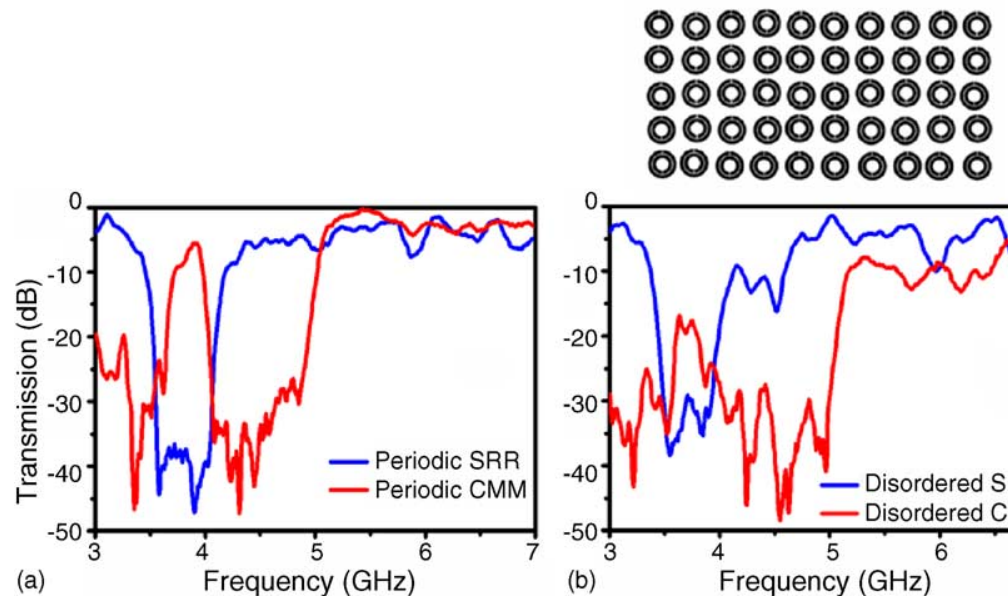

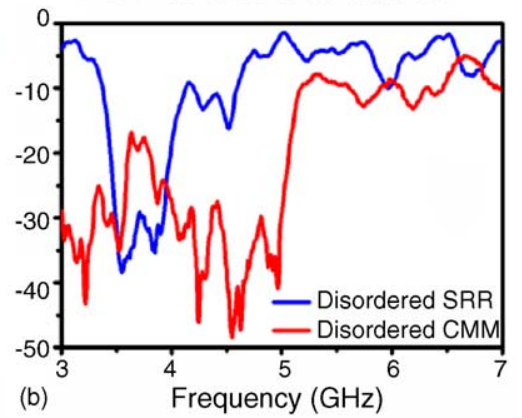

0000000000 0000000000 0000000000 0000000000 0000000000

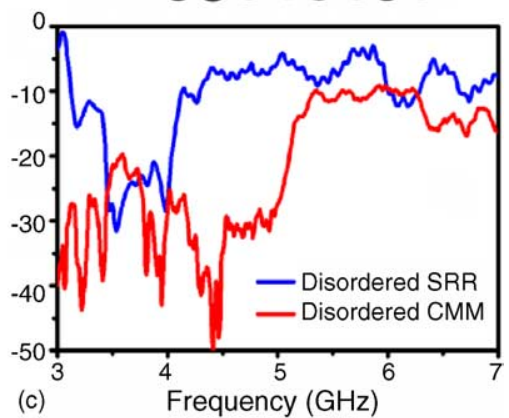

Fig. 5. (Top) The schematic view of weak (left) and strong (right) intralayer disorder of an SRR layer. (Bottom) The transmission spectra of (a) periodic, (b) weak disordered, and (c) strong disordered SRR structures (light lines) and the transmission spectra of corresponding CMM structures (dark lines), respectively.

We first consider the interlayer disorder for SRRonly medium. Fig. 4 shows the misalignment induced in lateral and longitudinal directions. For the longitudinal case, we consider weak and strong misalignments.

From Fig. 4, we conclude that the magnetic stop band of the SRR-only structure is quite persistent to misalignment, showing a slight shrinking in the bandwidth only.

The intralayer disorder is induced by displacing the SRR patterns within a layer randomly. Fig. 5 shows the transmission spectrum for two different disorder strengths (i.e., the maximum amount of displacement applied during randomizing the SRR coordinates. We take $\delta_{\mathrm{r}} \leq a / 10$ and $\delta_{\mathrm{r}} \leq a / 5$ as weak and strong disorders, where $\delta_{\mathrm{r}}$ is the displacement and $a$ is the intralayer period of SRRs. Evidently, the intralayer disorder has a significant effect. The strength and width of the magnetic resonance gap reduces. This indicates that the intralayer coupling of SRRs takes the prominent role in determining the collective SRR response.

\section{Conclusion}

In this report, we demonstrated experimentally that the electric response of SRRs is an integral part of the total response of CMM, and this must be taken into account in order to identify the left-handed transmission bands. We analyzed the left-handed behaviour of 1D and 2D CMM structures and demonstrated this behaviour by a refraction experiment. Then, we addressed the effect of disorder and misalignment on the transmission characteristics of stacking-type CMM structures. The intralayer disorder may degrade the magnetic response of SRRs and the associated lefthanded transmission band of CMMs. The SRR transmission spectrum is rather insensitive to misalignment effects, which is more likely to occur.

\section{References}

[1] V.G. Veselago, Electrodynamics of substances with simultaneously negative electrical and magnetic permeabilities, Sov. Phys. Usp. 10 (1968) 504.

[2] D.R. Smith, W.J. Padilla, D.C. Vier, S.C. Nemat-Nasser, S. Schultz, Composite medium with simultaneously negative permeability and permittivity, Phys. Rev. Lett. 84 (2000) 4184.

[3] R.A. Shelby, D.R. Smith, S. Schultz, Experimental verification of a negative index of refraction, Science 292 (2001) 77.

[4] J.B. Pendry, A.J. Holden, D.J. Robbins, W.J. Stewart, Low frequency plasmons from thin-wire structures, J. Phys. Condens. Matter 10 (1998) 4785.

[5] J.B. Pendry, A.J. Holden, D.J. Robbins, W.J. Stewart, Magnetism from conductors and enhanced nonlinear phenomena, IEEE Trans. Microwave Theory Tech. 47 (1999) 2075.

[6] T. Koschny, M. Kafesaki, E.N. Economou, C.M. Soukoulis, Effective medium theory of left-handed materials, Phys. Rev. Lett. 93 (2004) 107402.

[7] K. Aydin, K. Guven, M. Kafesaki, L. Zhang, C.M. Soukoulis, E. Ozbay, Experimental observation of true left-handed transmission peaks in metamaterials, Opt. Lett. 29 (2004) 2623.

[8] K. Aydin, K. Guven, C.M. Soukoulis, E. Ozbay, Observation of negative refraction and negative phase velocity in left-handed metamaterials, Appl. Phys. Lett. 86 (2005) 124102.

[9] K. Aydin, K. Guven, N. Katsarakis, C.M. Soukoulis, E. Ozbay, Effect of disorder on magnetic resonance band gap of split-ring resonator structures, Opt. Exp. 12 (2004) 5896. 